\title{
Exchange and Redistribution Dynamics of the Cytoskeleton of the Active Zone Molecule Bassoon
}

\author{
Shlomo Tsuriel, ${ }^{1}$ Arava Fisher, ${ }^{1}$ Nina Wittenmayer, ${ }^{2}$ Thomas Dresbach, ${ }^{2}$ Craig C. Garner, ${ }^{3}$ and Noam E. Ziv ${ }^{1}$ \\ ${ }^{1}$ The Rappaport Family Institute for Research in the Medical Sciences, Department of Physiology, Technion Faculty of Medicine, and the Lorry Lokey \\ Interdisciplinary Center for Life Sciences and Engineering, Haifa 32000, Israel, ${ }^{2}$ Institute of Anatomy and Cell Biology II, University of Heidelberg, \\ D-69120 Heidelberg, Germany, and ${ }^{3}$ Department of Psychiatry and Behavioral Science, Nancy Pritzker Laboratory, Stanford University, Palo Alto, \\ California 94304-5485
}

Presynaptic sites typically appear as varicosities (boutons) distributed along axons. Ultrastructurally, presynaptic boutons lack obvious physical barriers that separate them from the axon proper, yet activity-related and constitutive dynamics continuously promote the "reshuffling" of presynaptic components and even their dispersal into flanking axonal segments. How presynaptic sites manage to maintain their organization and individual characteristics over long durations is thus unclear. Conceivably, presynaptic tenacity might depend on the active zone (AZ), an electron-dense specialization of the presynaptic membrane, and particularly on the cytoskeletal matrix associated with the AZ (CAZ) that could act as a relatively stable "core scaffold" that conserves and dictates presynaptic organization. At present, however, little is known on the molecular dynamics of CAZ molecules, and thus, the factual basis for this hypothesis remains unclear. To examine the stability of the $\mathrm{CAZ}$, we studied the molecular dynamics of the major CAZ molecule Bassoon in cultured hippocampal neurons. Fluorescence recovery after photobleaching and photoactivation experiments revealed that exchange rates of green fluorescent protein and photoactivatable green fluorescent protein-tagged Bassoon at individual presynaptic sites are very low $(\tau>8 \mathrm{~h})$. Exchange rates varied between boutons and were only slightly accelerated by stimulation. Interestingly, photoactivation experiments revealed that Bassoon lost from one synapse was occasionally assimilated into neighboring presynaptic sites. Our findings indicate that Bassoon is engaged in relatively stable associations within the CAZ and thus support the notion that the CAZ or some of its components might constitute a relatively stable presynaptic core scaffold.

Key words: active zone; Bassoon; stability; FRAP; photoactivation; presynaptic tenacity

\section{Introduction}

The majority of presynaptic sites in the mammalian CNS appear as small varicosities (presynaptic boutons) distributed along axons. Synaptic connections arranged in this form (en passant synapses) allow a single axon to contact multiple postsynaptic cells as it courses through the neuropil. At the ultrastructural level, presynaptic boutons lack obvious physical barriers that separate their presynaptic structures from the cytoplasm and membrane of the axon proper (Shepherd and Harris, 1998). At the functional level, however, numerous processes, often associated with presynaptic activity, promote the dynamic "reshuffling" of presynaptic constituents and even their dispersal into flanking axonal segments. For example, presynaptic activity is associated with the mobilization of many, perhaps hundreds of synaptic vesicles (SVs) (Ryan and Smith, 1995; Kraszewski et al., 1996;

Received 0ct. 4, 2008; revised Nov. 6, 2008; accepted Nov. 18, 2008.

This work was supported by United States Israel Binational Science Foundation Grant 2003176 (C.C.G., N.E.Z.), Deutsche Forschungsgemeinschaft Grants Dr 373/3-1, Dr 373/3-2, and Dr 373/3-3 (T.D., N.E.Z.), and National Institutes of Health Grants NS39471 and NS353862 (C.C.G.). We are grateful to Larisa Goldfeld and Vladimir Lyakhov for their invaluable technical assistance.

Correspondence should be addressed to Noam E. Ziv, Network Biology Research Laboratories, Fishbach Building, Technion City, Haifa 32000, Israel. E-mail: noamz@netvision.net.il.

DOI:10.1523/JNEUROSCI.4777-08.2009

Copyright $\odot 2009$ Society for Neuroscience $\quad$ 0270-6474/09/290351-08\$15.00/0
Harata et al., 2001) with the rapid dispersal of presynaptic proteins such as Synapsin and Rab3 (Chi et al., 2001, 2003; Star et al., 2005) and with the deposition of SV membrane proteins on the axonal plasma membrane, some of which wander away, only to reappear in nearby boutons (Sankaranarayanan and Ryan, 2000, 2001; Li and Murthy; 2001; Fernández-Alfonso et al., 2006; Wienisch and Klingauf 2006). At longer timescales, packets of SVs (Darcy et al., 2006), active zonal material (Krueger et al., 2003), and synaptic matrix proteins (Tsuriel et al., 2006) migrate along the axon from one presynaptic site to another. Despite these dynamics and the lack of obvious physical barriers, mammalian CNS presynaptic sites do persist for long periods (De Paola et al., 2006; Stettler et al., 2006) and (as far as we know) do not eventually equilibrate and become identical in size, composition, or function (Murthy et al., 1997; Staple et al., 2000; Geracitano et al., 2007). How do synapses maintain their individual functionalities? What is the basis for the tenacity exhibited by the minute and dynamic presynaptic specialization?

The presynaptic plasma membrane contains an electrondense thickening, known as the active zone (AZ), which contains a dense meshwork of structural proteins known as the cytoskeletal matrix associated with the AZ (CAZ) (Schoch and Gundelfinger, 2006). The CAZ is remarkably resistive to chemical extraction procedures, and many of its constituents seem to be 
engaged in high-affinity interactions. These characteristics suggest that the CAZ may function as a "core scaffold" that specifies and maintains the position of presynaptic, cytosolic, and membranal molecules while providing a positional reference and "anchoring" site for molecular mesh works that cluster SVs at presynaptic boutons. It is therefore conceivable that presynaptic tenacity is based to a large degree on the tenacity of the CAZ.

Somewhat unexpectedly, a recent study revealed that the CAZ protein Munc13-1 exhibits relatively rapid exchange rates ( $\tau$ of $\approx 1 \mathrm{~h}$ ), which could indicate that the CAZ is more dynamic than previously conceived (Kalla et al., 2006). Munc13-1, however, has also been shown to dynamically translocate to the plasma membrane in response to phorbol-ester binding (Betz et al., 1998), which indicates that Munc13-1 is not as tightly associated with the AZ as other CAZ molecules may be. Indeed, the CAZ contains additional components, some of which seem less likely to exhibit such rapid dynamics. These include Piccolo and Bassoon, very large and related proteins of 560 and $420 \mathrm{kDa}$, respectively (tom Dieck et al., 1998; Fenster et al., 2000). These molecules are found in molecular complexes that can be also immunoprecipitated together with postsynaptic proteins (Husi et al., 2000), are not lost in conditions that cause the dissociation of many presynaptic AZ proteins (Phillips et al., 2001), and, unlike other AZ molecules, do not change their ultrastructural distribution after stimulation (Tao-Cheng, 2006). We therefore set out to examine the cellular dynamics of one of these proteins, namely Bassoon, to determine whether it is a significantly more stable CAZ component and thus lend credence to the idea that the CAZ or some of its components might constitute a relatively stable, presynaptic core scaffold.

\section{Materials and Methods}

Cell culture. Hippocampal cell cultures were prepared from 1- to 2-d-old Sprague Dawley rats as described previously (Tsuriel et al., 2006). Neurons were used for experiments $14-25 \mathrm{~d}$ after plating. Reagents were procured from the following sources: 6-cyano-7-nitroquinoxaline-2,3dione (CNQX) was from Tocris Bioscience; 2-amino-5phosphonopentanoic acid (AP-5) was from Sigma; and FM4-64 (N-(3triethylammoniumpropyl)-4-( $p$-dibutylaminostyryl)pyridinium, dibromide) was from Invitrogen.

DNA constructs and expression. The GFP:Bsn95-3938 construct used here was described previously (Dresbach et al., 2003). PAGFP:Bsn953938 was prepared by substituting green fluorescent protein (GFP) with photoactivatable GFP (PAGFP) (a generous gift from Dr. LippincottSchwartz, National Institute of Child Health and Human Development, Bethesda, MD). In both constructs, Bsn95-3938 expression was driven by a cytomegalovirus promoter. Expression of fusion proteins was performed by calcium phosphate transfection on days 7-10 in vitro as described previously (Bresler et al., 2004). Coexpression of PAGFP:Bsn953938 and cyan fluorescent protein (CFP) was performed in a similar manner using a 9:1 mixture of the two vectors precipitated by ethanol and resuspended in distilled water.

Microscopy. Fluorescence and differential interference contrast (DIC) images were acquired using a custom-designed confocal laser scanning microscope (Tsuriel et al., 2006) using a $40 \times, 1.3$ numerical aperture Fluar objective. CFP and enhanced GFP (EGFP)/PAGFP/FM4-64 were excited using the 457 and $488 \mathrm{~nm}$ lines of an argon laser (JDS Uniphase), respectively. Fluorescence emissions were read using 467-493 (CFP), 500-545 (EGFP/PAGFP) nm bandpass, and >630 nm (FM4-64) longpass filters (Chroma Technology). Photoactivation was performed using a $405 \mathrm{~nm}$ diode laser (Coherent). Time-lapse recordings were performed by averaging three to six frames at two to three focal planes spaced 1-1.5 $\mu \mathrm{m}$ apart. All data were collected at a resolution of $640 \times 480$ pixels, at 12 bits/pixel, with the confocal aperture fully open. Some long-term fluorescence recovery after photobleaching (FRAP) and all photoactivation experiments were performed without replacing the growth media (no perfusion), in a sterile atmospheric mixture of $5 \% \mathrm{CO}_{2}$ and $95 \%$ air. The majority of FRAP experiments were performed in a laminar flow perfusion insert mounted in a modified heated chamber (Warner Instruments) and perfused with preheated Tyrode's saline solution (in mм: 119 $\mathrm{NaCl}, 2.5 \mathrm{KCl}, 2 \mathrm{CaCl}_{2}, 2 \mathrm{MgCl}_{2}, 25$ HEPES, and 30 glucose, $\mathrm{pH} 7.4$ ) containing CNQX $(10 \mu \mathrm{M})$ and AP-5 $(50 \mu \mathrm{M})$. Data were collected at predetermined time intervals, with focal drift corrected before collecting each image stack using the microscope systems "autofocus" feature. Stimulation, FM4-64 labeling, photobleaching, and photoactivation were performed as described previously (Tsuriel et al., 2006).

Retrospective immunohistochemistry. FRAP experiments were followed by fixation in $4 \%$ formaldehyde and $120 \mathrm{~mm}$ sucrose in PBS for $20 \mathrm{~min}$, followed by permeabilization for $10 \mathrm{~min}$ in fixative solution containing $0.25 \%$ Triton X-100 (Sigma). Cells were washed three times in PBS, incubated in $10 \%$ bovine serum albumin (BSA) for $1 \mathrm{~h}$ at $37^{\circ} \mathrm{C}$, and incubated overnight at $4^{\circ} \mathrm{C}$ with primary antibodies (guinea pig antiProSAP2) (Bresler et al., 2004) in PBS and 1\% BSA. Cells were rinsed three times for 10 min with PBS and incubated for $1 \mathrm{~h}$ at room temperature with secondary antibodies [cyanine 5 (Cy5) conjugated donkey anti-guinea pig (Jackson ImmunoResearch)] in PBS and 1\% BSA. The cells were rinsed again with PBS, mounted, and imaged immediately. Location of photobleached boutons in immunolabeled specimens was performed as described previously (Bresler et al., 2004). Images were collected by averaging three frames at six sections spaced $0.5 \mu \mathrm{m}$ apart with the confocal aperture nearly fully closed. Cy5 fluorescence was recorded at $633 \mathrm{~nm}$ excitation (helium-neon 633 line)/>640 emission.

Image analysis. All data analysis was performed using software (OpenView) written for this purpose by one of the us (N.E.Z.). Analysis was performed on maximal intensity projections of $Z$-section stacks. Intensities of fluorescent puncta were measured as mean pixel values in $10 \times$ 10 pixel rectangular regions centering on them. FRAP data normalization, correction for ongoing photobleaching, and fitting of recovery curves were performed as described previously (Tsuriel et al., 2006). Normalization of quantitative photoactivation data were performed as follows: Recovery $=(F t-F b) /(F a-F b)$, where $F b$ is the fluorescence of a PAGFP: Bsn95-3938 punctum before photoactivation, $\mathrm{Fa}$ is the fluorescence of the same punctum immediately after photoactivation, and $F t$ is the fluorescence of that punctum at time $t$.

Final images were prepared using Adobe Photoshop and Microsoft PowerPoint.

\section{Results}

To study the rates at which Bassoon is lost from and reincorporated into the CAZ of individual presynaptic sites, we expressed a GFP-tagged variant of Bassoon (GFP:Bsn95-3938) in hippocampal neurons obtained from newborn rats and grown in culture for at least 2 weeks. This slightly truncated variant of Bassoon was shown previously to be targeted correctly to presynaptic sites (Dresbach et al., 2003, 2006) and was used to study the recruitment of Bassoon to new presynaptic sites (Bresler et al., 2004). When expressed in cultured hippocampal neurons, GFP:Bsn953938 exhibits a punctate distribution pattern (Fig. 1). In these rather mature preparations, most GFP:Bsn95-3938 puncta are relatively stable and correspond to functional presynaptic sites (Bresler et al., 2004), although some smaller (and dimmer) mobile puncta are also observed.

The loss and reincorporation rates of GFP:Bsn95-3938 at individual presynaptic sites were measured by FRAP. To that end, neurons expressing GFP:Bsn95-3938 were placed in a laminar flow chamber and maintained at $33-35^{\circ} \mathrm{C}$ in a physiological solution. After collecting baseline images, three to five fluorescent puncta were selectively bleached by high-intensity $488 \mathrm{~nm}$ laser light (Tsuriel et al., 2006), after which fluorescence recovery was monitored by collecting image stacks (two to three sections) at rates of one image every $5-10 \mathrm{~min}$ for $\sim 2 \mathrm{~h}$ (Fig. $2 A, B$ ). To verify that the photobleached puncta represented functional presynap- 

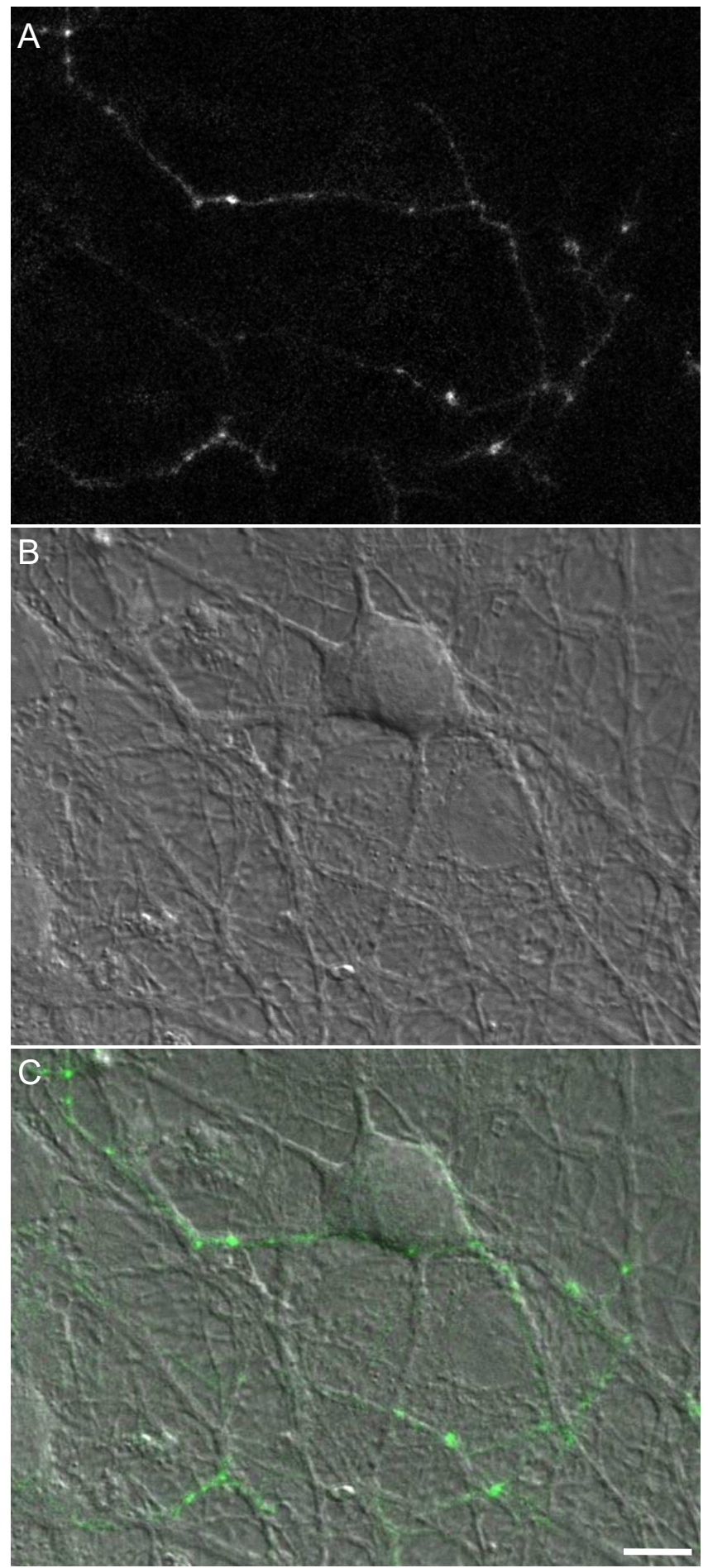

Figure 1. Expression of GFP:Bsn95-3938 in cultured hippocampal neurons. $\boldsymbol{A}$, Fluorescence image of an axon belonging to a neuron expressing GFP:Bsn95-3938. $\boldsymbol{B}$, DIC image of the same region. C, Fluorescence image overlaid on the DIC image. Note the bright puncta at intersection points between axonal arbors and dendrites. Most such puncta represent functional presynaptic sites; $14 \mathrm{~d}$ in vitro. Scale bar, $10 \mu \mathrm{m}$.

tic sites and that this functionality was retained after the photobleaching procedure, field stimulation $(30 \mathrm{~s}$ at $10 \mathrm{~Hz})$ was used to label all boutons with the fluorescent functional endocytosis marker FM4-64. Only GFP:Bsn95-3938 puncta that exhibited a capacity to uptake FM4-64 after the labeling procedure and subsequently release it after a second stimulation episode (120 s at 10 $\mathrm{Hz}$ ) were included in our analysis (Fig. 2C).
As shown in Figure 2, fluorescence recovery varied significantly from one bouton to another. Whereas fluorescence recovery was quite significant in some boutons (for example, bouton 1 in Fig. 2), in others it was much slower (boutons 2, 3). Because the imaging procedure itself was associated with some degree of photobleaching, we corrected the recovery data of the photobleached boutons according to the slight photobleaching exhibited by "naive" boutons in the same fields of view (Tsuriel et al., 2006). Even with this correction, however, fluorescence recovery remained variable.

The recovery process was also qualitatively variable: a minority (approximately one-quarter) of photobleached boutons exhibited gradual and monotonic recovery, but the majority exhibited somewhat erratic recovery patterns, occasionally involving stepwise changes in fluorescence (supplemental Fig. 1, available at www.jneurosci.org as supplemental material). However, when data from all experiments ( 26 boutons, 14 fields of view, 5 separate experiments) were pooled together, an apparently smooth recovery curve emerged (Fig. $3 A$ ). Assuming the existence of two GFP:Bsn95-3938 pools with fast and slow exchange rates, this curve could be fit to two exponentials with time constants of 5 and $245 \mathrm{~min}$ and pool ratios of $\sim 1: 4$, respectively (Tsuriel et al., 2006). An extrapolation of this curve indicates that, on average, individual presynaptic sites exchange their entire pool of GFP: Bsn95-3938 every 16-18 h (Fig. 3B).

Several reservations are important to mention. First, the time constant calculated for the slowly exchanging (and major) GFP: Bsn95-3938 pool is based on an extrapolation of data obtained for the first $2 \mathrm{~h}$ of a much longer process and thus is quite sensitive to relatively small errors in measured (and corrected) recovery rates, mainly those made at later times (near the $2 \mathrm{~h}$ mark). It thus should be acknowledged that this time constant is probably somewhat inaccurate. Second, the time constants above represent only average values; individual boutons exhibited a range of recovery rates, as evidenced by the error bars in Figure $3 \mathrm{~A}$. In fact, approximately one-quarter of the boutons exhibited very little $(<30 \%)$ recovery over $2 \mathrm{~h}$, whereas the remainder exhibited intermediate $(30-70 \%)$ to substantial $(>70 \%)$ recovery over the same period (Fig. $3 C$ ). In these populations, the mean slow time constants were $>20,3.7$, and $1.3 \mathrm{~h}$, respectively.

Variability of exchange rates at different boutons occurred across different experiments, different cells in the same dish, and different boutons along the same axons (although to a somewhat lesser degree). We did not find consistent relationships between GFP:Bsn95-3938 exchange rates and initial GFP:Bsn95-3938 puncta size (data not shown). To explore potential relationships between GFP:Bsn95-3938 exchange rates and SV recycling, we performed additional FRAP experiments ending with FM4-64 labeling by 2 - or 120 -s-long stimuli trains at $10 \mathrm{~Hz}$, but no consistent relationships were found between FM4-64 labeling and GFP:Bsn95-3938 exchange rates at the same boutons (data not shown). We also explored the possibility that some of the variability might have stemmed from the inclusion of presynaptic sites lacking postsynaptic counterparts ("orphan synapses") (Krueger et al., 2003) in our analysis. To that end, we performed an additional set of FRAP experiments, after which the cells were fixed and labeled with antibodies against the postsynaptic density protein ProSAP2 (supplemental Fig. 2, available at www. jneurosci.org as supplemental material). Of the 17 individually photobleached GFP:Bsn95-3938 puncta identified with absolute certainty, only two (11\%) were not juxtaposed against a cluster of ProSAP2 (10 fields of view, 4 separate experiments). The recovery exhibited by these boutons was not remarkably different from the 

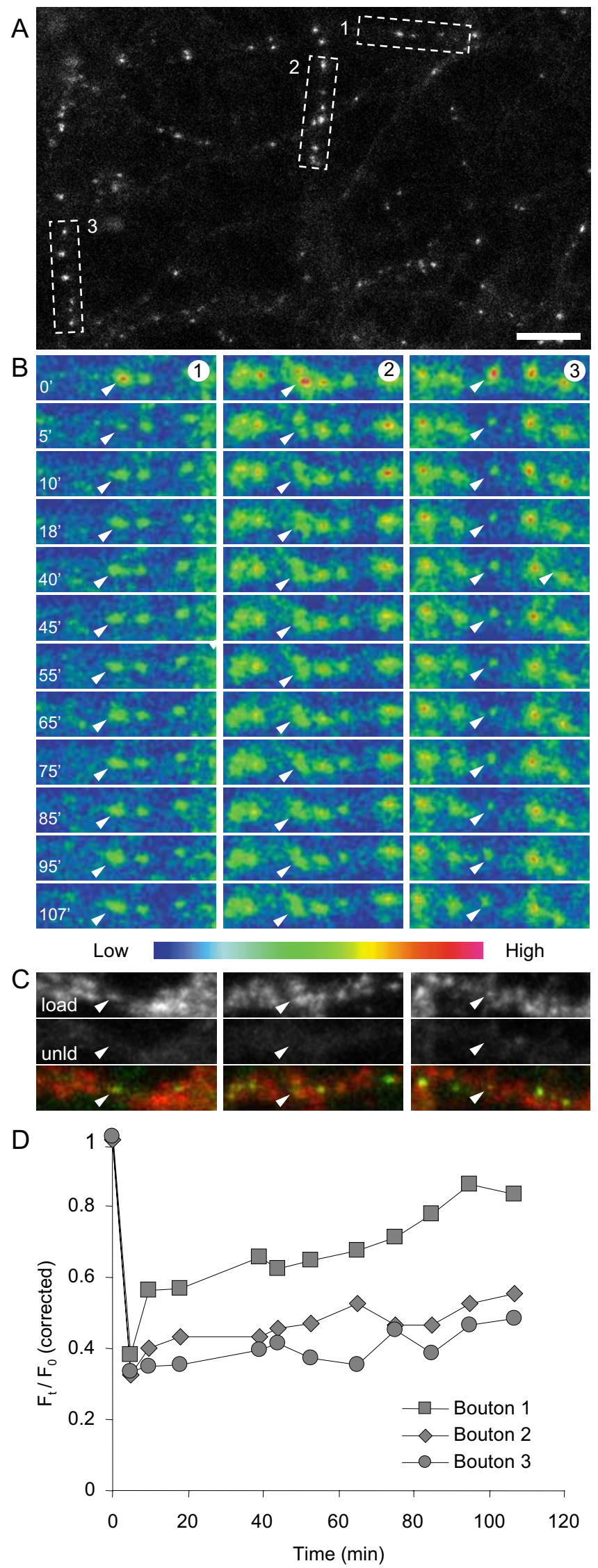

Figure 2. Loss and reincorporation rates of GFP:Bsn95-3938 at individual presynaptic boutons. $A$, Axons of neurons expressing GFP:Bsn95-3938. Three GFP:Bsn95-3938 puncta, one in each rectangular region, were selected for photobleaching. Scale bar, $10 \mu \mathrm{m}$. $\boldsymbol{B}$, The three puncta were selectively photobleached by high-intensity laser light (arrowheads), and fluores- other 15. Furthermore, the distribution of recoveries exhibited by ProSAP2-associated GFP:Bsn95-3938 puncta was similar to that observed for the entire population (supplemental Fig. $2 C$, available at www.jneurosci.org as supplemental material). These findings thus do not support the possibility that the variability in recovery rates we observed stemmed from the inclusion of orphan presynaptic sites in our analysis.

If Bassoon is part of a relatively stable presynaptic scaffold, it might be expected that the interactions it is engaged in would be rather indifferent to the intense dynamics induced by activity. We thus compared, in pairwise manner, Bassoon exchange rates in preparations in which spontaneous activity was blocked by application of CNQX $(10 \mu \mathrm{M})$ and AP-5 $(50 \mu \mathrm{M})$, with exchange rates in preparations stimulated periodically in the presence of the same antagonists $(20 \mathrm{~s}$ at $20 \mathrm{~Hz}$, every $3 \mathrm{~min}$; stimulation beginning immediately after collecting the first post-photobleach images). Here too we observed significant variability in fluorescence recovery patterns and rates ( $n=44$ and 29 boutons; nonstimulated and stimulated, respectively, 17 separate experiments), yet when average recovery rates were plotted against each other (Fig. $3 D$ ), a small but statistically significant increase in fluorescence recovery rates was observed for the stimulated population $(p=$ 0.036 , ANOVA with repeated measures). In addition, stimulation was associated with a shift in the bouton population toward more substantial recovery over the same period (Fig. 3D, inset). Thus, Bassoon exchange rates are not entirely indifferent to presynaptic activation, but the effects are relatively modest.

The FRAP experiments described so far consistently indicated that the exchange time constant for the slowly exchanging GFP: Bsn95-3938 pool is on the order of several hours. However, as discussed above, these FRAP-based estimates were probably somewhat inaccurate. We thus used a second method to measure the kinetics of the slowly exchanging GFP:Bsn95-3938, namely fluorescence recovery after photoactivation (FRAPA). To that end, we substituted the EGFP moiety of GFP:Bsn95-3938 with PAGFP (Patterson and Lippincott-Schwartz, 2002) and transfected neurons with this fusion protein. Because PAGFP is practically invisible before photoactivation, CFP was cotransfected with PAGFP:Bsn95-3938 to allow us to locate and select putative presynaptic sites for photoactivation (Fig. 4A) (Tsuriel et al., 2006). Spatially confined, high-intensity illumination at $405 \mathrm{~nm}$ was then used to photoactivate PAGFP:Bsn95-3938 at these sites, and the decay of photoactivated PAGFP:Bsn95-3938 fluorescence was then followed by time-lapse microscopy.

Although FRAPA and FRAP are conceptually equivalent, photobleaching associated with time-lapse imaging breaks the symmetry of these two methods: in FRAP, photobleaching leads to an underestimation of recovery rates, whereas in FRAPA, it leads to an overestimation of exchange rates. Furthermore, the bleaching rate of photoactivated PAGFP:Bsn95-3938 was found to be approximately twice as large as that of GFP:Bsn95-3938 (supplemental Fig. 3, available at www.jneurosci.org as supple-

$\leftarrow$

cence recovery at these sites was subsequently followed by time-lapse imaging. Fluorescence levels shown in false color according to color scale near the bottom. $\boldsymbol{C}$, At the end of the experiment, presynaptic boutons were labeled with FM4-64 by field stimulation ("load"; $30 \mathrm{~s}$ at 10 $\mathrm{Hz}$ ), followed by unloading ("unld"; $120 \mathrm{~s}$ at $10 \mathrm{~Hz}$ ) to verify the functionality of the photobleached GFP:Bsn95-3938. Note that all three photobleached puncta (green channel, bottom panels) exhibited a capacity for both FM4-64 endocytosis and exocytosis. D, Fluorescence recovery time course for the three photobleached puncta, after correcting for ongoing photobleaching (see Results). Note that whereas bouton 1 exhibited significant recovery, boutons 2 and 3 exhibited very little recovery over the same period. 

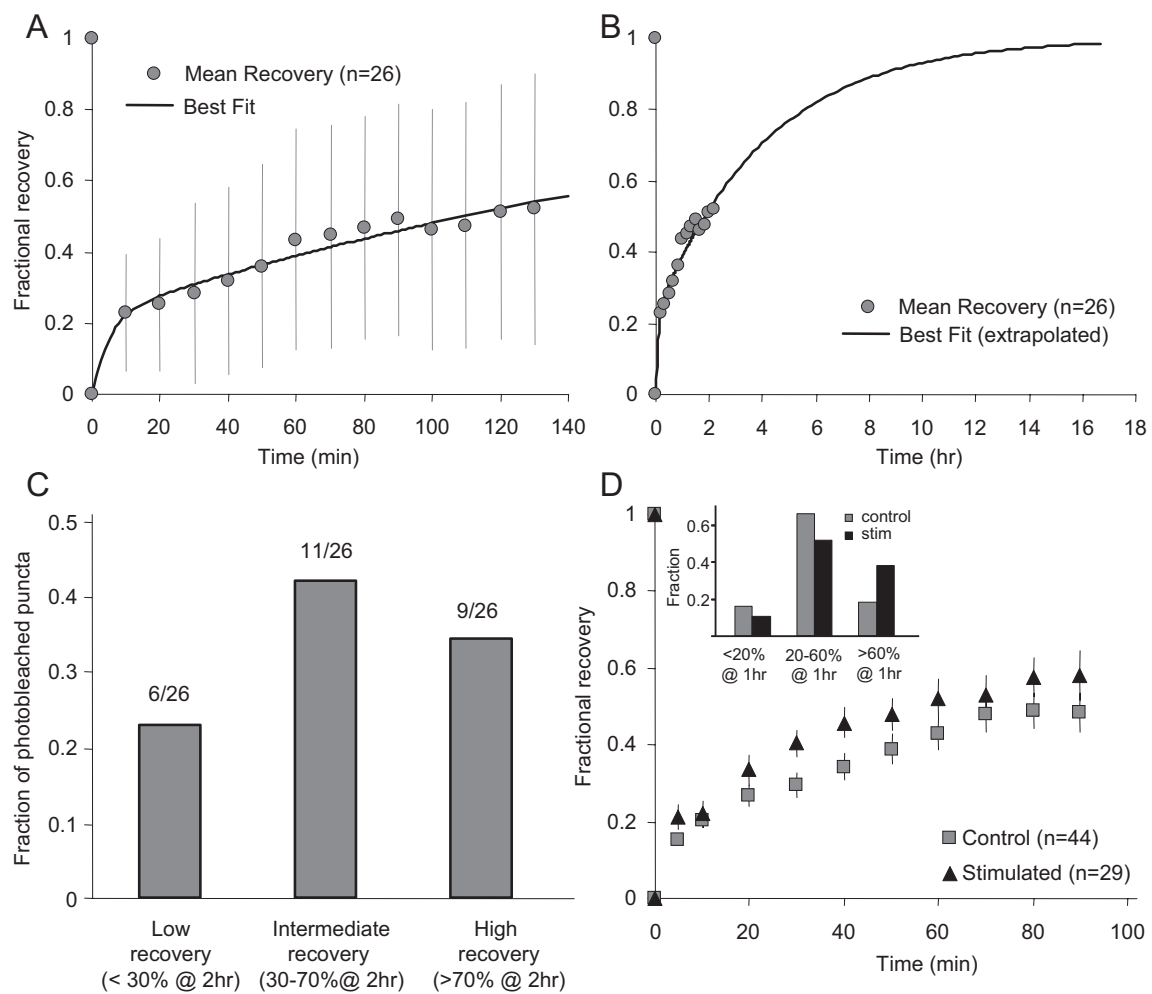

Figure 3. GFP:Bsn95-3938 recovery rates are highly variable. $A$, Mean recovery time course for all photobleached GFP:Bsn953938 puncta. Note the variability of fluorescence recovery rates as evident from the SDs (errors bars). The data were fit according to a model that assumed two GFP:Bsn95-3938 pools with different recovery kinetics (Tsuriel et al., 2006). The best fit was obtained with time constants of $5 \mathrm{~min}$ (fast pool) and $245 \mathrm{~min}$ (slow pool) and a fast/slow pool ratio of $\sim 1: 4$, respectively. $\boldsymbol{B}$, Extrapolation of the best fit curve indicates that, on average, the entire content of GFP:Bsn95-3938 at individual presynaptic boutons is replaced every $16-18 \mathrm{~h}$. C, Photobleached puncta grouped according to degree of recovery observed after $2 \mathrm{~h}$. D, Recovery of fluorescence in boutons stimulated at $20 \mathrm{~Hz}$ for $20 \mathrm{~s}$ every 3 min compared with controls in which no stimulation was performed (means \pm SEM). Recovery rates in the stimulated population were, on average, slightly faster than those of the control population. Stimulation was also associated with a shift in the bouton population toward boutons that exhibited greater recovery over a $1 \mathrm{~h}$ period (inset).

mental material). Finally, in FRAP, errors arising from photobleaching are more significant near the end of the recovery phase (which is critical for correct estimations of slow exchange rates), whereas in FRAPA, such errors are extremely significant at initial phases of recovery (this is demonstrated in an interactive spreadsheet provided as supplemental data, available at www. jneurosci.org as supplemental material). We thus optimized the experimental protocol to match the advantages and disadvantages of FRAPA. Specifically, we used FRAPA to measure the exchange kinetics of the slowly exchanging (PA)GFP:Bsn95-3938 pool by following the decay of photoactivated PAGFP:Bsn953938 fluorescence at very low sampling rates (one image per hour) for 13-20 h, purposely ignoring the decay kinetics during the first 1-2 h. As shown in Figure $4 B$, fluorescence decay occurred over many hours. When data from all experiments were pooled together ( 45 boutons, 8 cells, 5 separate experiments), the mean decay plots could be fit to two exponentials (Fig. 4C). The time constant for the "fast" pool is of no significance because the initial recovery period was undersampled for reasons explained above. However, the sampling rates and durations were well suited for measuring the exchange rates of the slowly exchanging pool, and thus the time constant measured for this component, $501 \mathrm{~min}(>8 \mathrm{~h})$, is quite reliable. When bringing into account the fact that FRAPA-based measurements inherently overestimate exchange rates, these experiments strongly confirm and extend the previous conclusion (Fig. 3) that the loss and reincorporation rates of presynaptic bassoon are indeed very slow. It should be noted that significant variability in the exchange rates of different boutons was also evident in FRAPA experiments (Fig. 4D), although it was slightly less pronounced, probably as a result of the low sampling rates and the fact that no compensation for ongoing bleaching was performed.

Previous studies have shown that some presynaptic proteins are continuously exchanged among nearby presynaptic boutons (Sankaranarayanan and Ryan, 2000, 2001; Li and Murthy, 2001; Darcy et al., 2006; Fernández-Alfonso et al., 2006; Tsuriel et al., 2006; Wienisch and Klingauf 2006) (for review, see Staras, 2007). Are CAZ molecules interchanged as well? Can Bassoon lost from one presynaptic locus migrate to nearby presynaptic sites and assimilate into the CAZ at these loci? To examine this possibility, we photoactivated PAGFP:Bsn95-3938 in small groups of presynaptic boutons (Fig. 5C,D) and followed the redistribution of photoactivated PAGFP:Bsn95-3938 by time-lapse imaging (Fig. $5 F$ ). In 8 of 14 experiments (7 different preparations), photoactivated PAGFP:Bsn95-3938 was clearly observed to accumulate at AZs residing outside the originally photoactivated region (Fig. $5 F$ ). We were interested to determine the mode of PAGFP:Bsn95-3938 accumulation at these sites: did fluorescence increase gradually and monotonically, or was material shuttled to these sites in the form of mobile packets as shown previously to occur during synaptogenesis (Krueger et al., 2003; Shapira et al., 2003; Bresler et al., 2004; Dresbach et al., 2006; Tao-Cheng, 2007)? Indeed, mobile packets of photoactivated PAGFP:Bsn95-3938 were occasionally observed to move inside and away from the photoactivation regions (for example, see $t=68$ in Fig. $5 F$ and supplemental Fig. 1, available at www.jneurosci.org as supplemental material). To that end, we repeated these experiments at higher temporal resolutions better matched to axonal transport processes (two to four images per minute). However, at these sampling rates, photobleaching reduced the fluorescence of photoactivated PAGFP: Bsn95-3938 to very low levels within 10-20 min (supplemental Fig. 3, available at www.jneurosci.org as supplemental material), and ultimately we were not able to determine with absolute certainty that such packets were a major source of photoactivated PAGFP:Bsn95-3938 that accumulated at nonphotoactivated AZs ( $>15$ separate experiments). We thus conclude that, in common with other presynaptic (and postsynaptic) molecules studied so far, Bassoon seems to be interchanged among nearby synapses, although the mode of interchanging remains uncertain.

\section{Discussion}

The molecular dynamics associated with SV release on the one hand and the lack of obvious structural barriers that confine presynaptic components to presynaptic compartments on the 


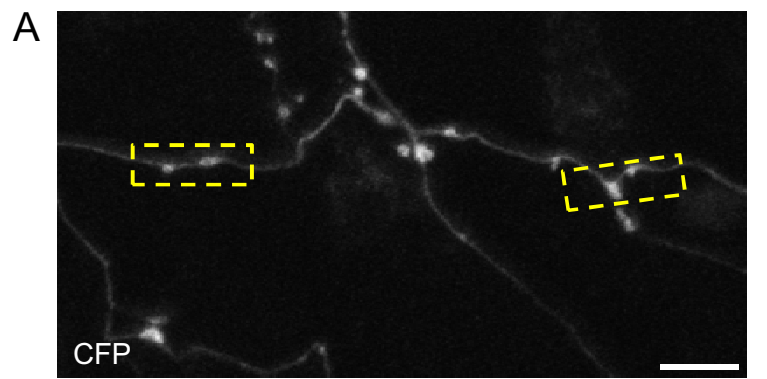

B Before photoactivation

After first photoactivation
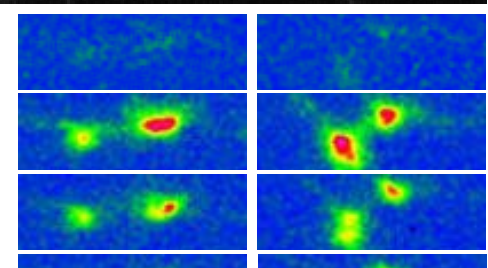

$1 \mathrm{hr}$

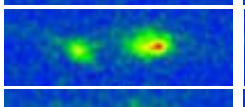

$2 \mathrm{hrs}$

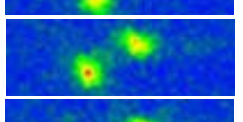

$3 \mathrm{hrs}$
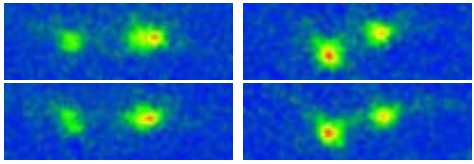

$4 \mathrm{hrs}$
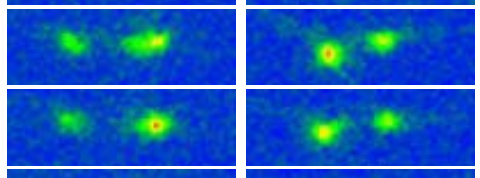

$8 \mathrm{hrs}$

$10 \mathrm{hrs}$

$12 \mathrm{hrs}$
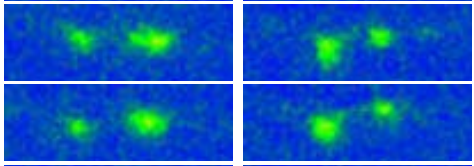

$13.4 \mathrm{hrs}$
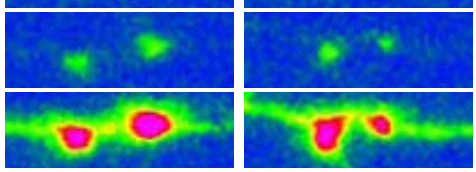

Second photoactivation

C
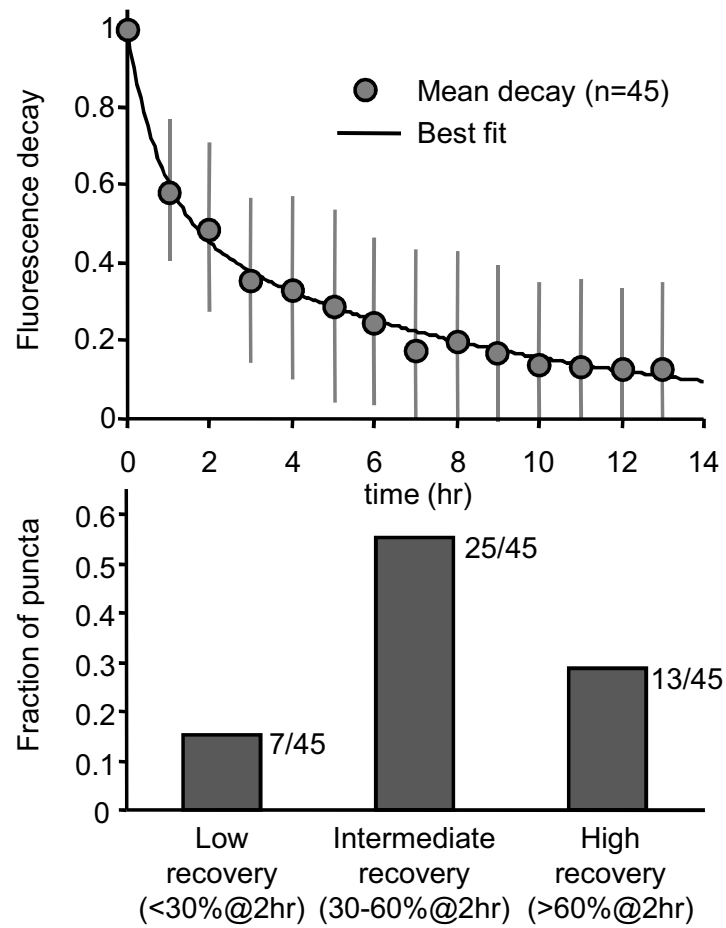

Figure 4. Loss and reincorporation rates of PAGFP:Bsn95-3938 measured by FRAPA. A, Axons of neurons expressing both CFP and PAGFP:Bsn95-3938 (CFP channel is shown). Several rectangular regions containing distinct varicosities were selected for photoactivation. Scale bar, other would seem to seriously challenge the stability and tenacity of the presynaptic bouton. In this study, we examined the molecular dynamics exhibited by Bassoon, one of the largest CAZ molecules, to examine the possibility that the CAZ or some of its components might function as a relatively stable "core scaffold" that defines and maintains the characteristics and organization of the presynaptic site.

Both FRAP and photoactivation experiments strongly indicate that the exchange rates of (PA)GFP:Bsn95-3938 at individual presynaptic sites are relatively low. The actual estimates of the slow component exchange rate differed somewhat in FRAP and FRAPA experiments. However, for reasons explained above, the FRAPA experiments were much better suited for estimating these slow exchange rates. Furthermore, because FRAPA measurements inherently overestimate exchange rates, our conclusion that Bassoon exchange rates are relatively slow (time constant $>8$ h) seems to be well founded. In fact, to the best of our knowledge, these exchange rates are the lowest reported to date for any (pre) synaptic protein. We stress, however, that this figure should not be taken too literally for several reasons: (1) significant variability was observed in the recovery rates of individual boutons; (2) the fusion proteins used here lack the first 94 aa of native Bassoon (and the myristolization sites found within this segment); and (3) the fusion protein has a $30 \mathrm{kDa}$ protein (GFP or PAGFP) appended to its $\mathrm{N}$ terminus. Finally, this number is undoubtedly affected by the preparation used here. Aside from the fact that the experiments were performed in a reduced system (cell culture), they were performed in neurons that are relatively immature (2-4 weeks in vitro) compared with those in the mature rat brain. Given a recent in vivo study showing that the exchange dynamics of a postsynaptic protein (PSD-95) decrease with age (Gray et al., 2006), the exchange rates reported here are likely to overestimate the exchange rates of Bassoon in the mature brain. However, even if the rates reported here are overestimates, they are still several orders of magnitude slower than the timescales typically associated with SV recycling, as well as Synapsin I, actin, Rab3, and SV protein redistribution, and suggest that at least some CAZ components are engaged in relatively stable associations. This conclusion is also supported by our finding that GFP:Bsn95-3938 exchange rates are only mildly affected by strong stimulation (Fig. 3D), as would be expected for a relatively stable protein complex whose protein-protein interactions are not significantly weakened by activity and the SV recycling dynamics it entails. Thus, at least some components of the CAZ appear to be part of a relatively stable scaffold that might serve metaphorically as "an island of stability in a sea of change" that defines, conserves, and maintains the location, size, and individual characteristics of each presynaptic site, at least on a $5-10 \mathrm{~h}$ timescale.

The relatively slow exchange rates of Bassoon are consistent with the possibility that these reflect rates of Bassoon degradation

$\leftarrow$

$10 \mu \mathrm{m} . \boldsymbol{B}$, Boutons that were selectively photoactivated by brief, high-intensity illumination at $405 \mathrm{~nm}$ (regions enclosed in yellow rectangles in $\boldsymbol{A}$ ). Fluorescence decay at these sites was subsequently followed by time-lapse imaging. Full-field photoactivation performed at the end of the experiments greatly increased fluorescence levels at these sites, indicating that the reduction in fluorescence observed during the experiments was not entirely attributable to photobleaching but was, at least in part, attributable to the dissociation of photoactivated PAGFP: Bsn95-3938 and replacement with nonphotoactivated PAGFP:Bsn95-3938. Fluorescence levels shown in false color according to color scale in Figure 2.C, Mean fluorescence decay time course for all photoactivated PAGFP:Bsn95-3938 puncta and fit to two exponentials. D, Photoactivated puncta grouped according to the degree of recovery observed after $2 \mathrm{~h}$. 

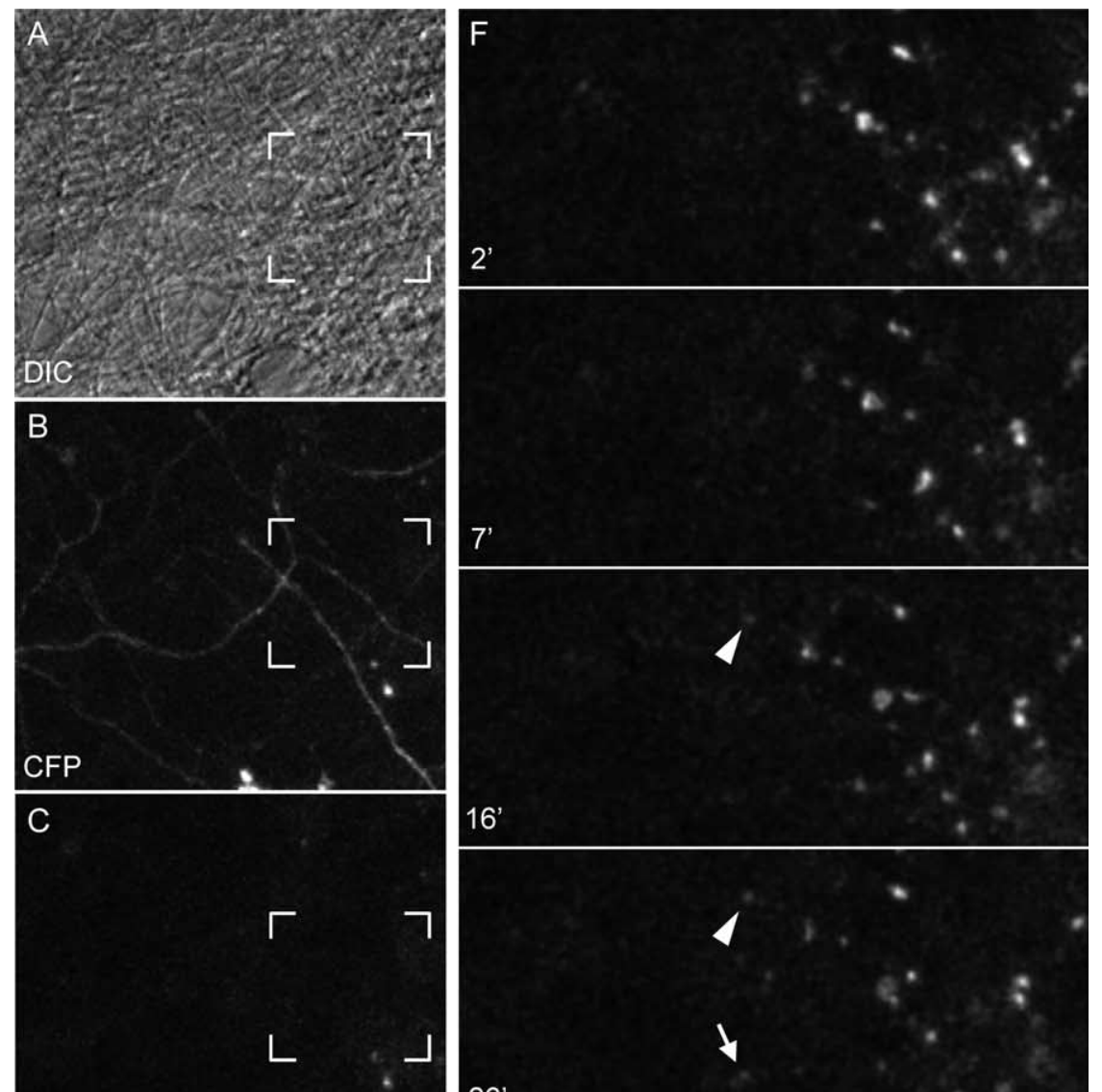

2
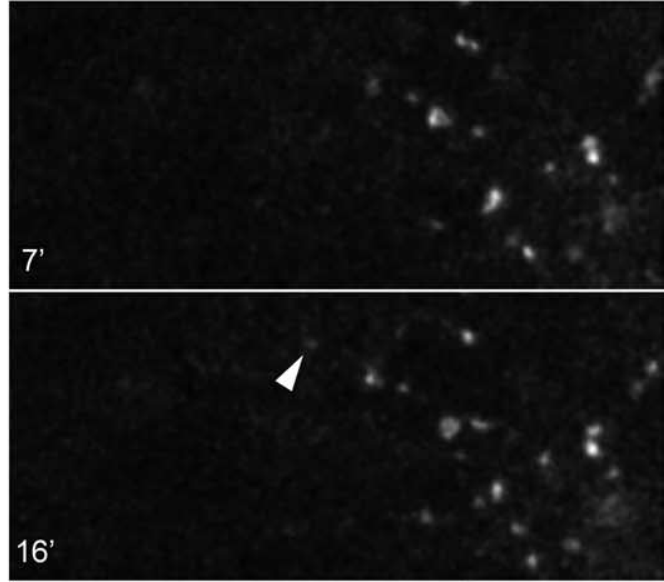

Before

photoactivation

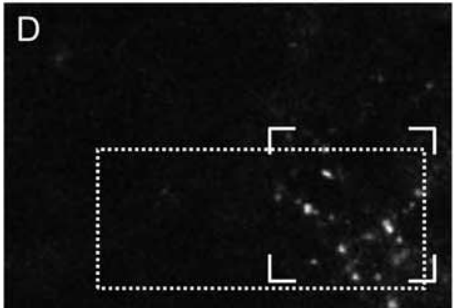

Restricted photoactivation

$\mathrm{t}=2$

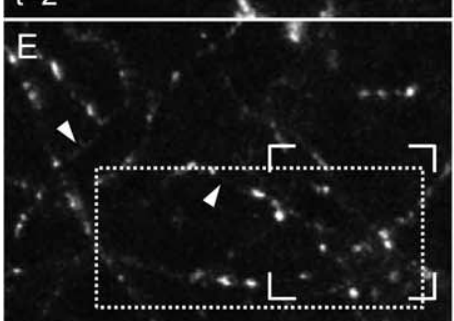

*

Full photoactivation $t=181^{\prime}$

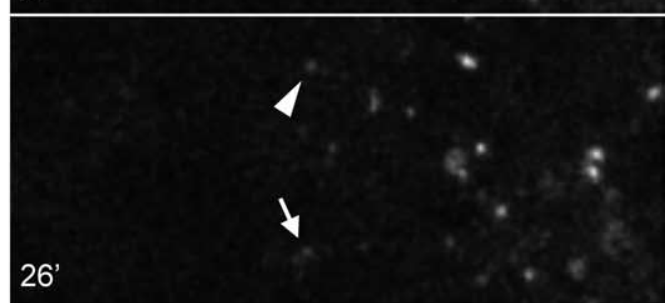

68
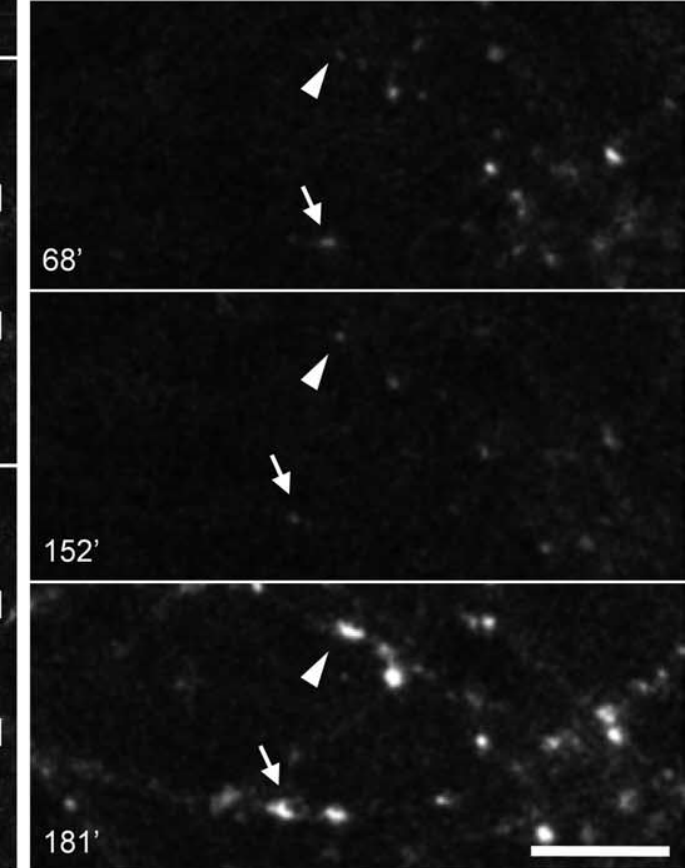

Figure 5. Redistribution of PAGFP:Bsn95-3938 among nearby synapses. Image of axons from neurons expressing both CFP and PAGFP:Bsn95-3938. A, DIC image. B, CFP channel. C, PAGFP:Bsn95-3938, before photoactivation. D, Same region as in A-C shortly after selective photoactivation of region enclosed in reticule. $\boldsymbol{E}$, Same region after nonselective photoactivation of entire field at the end of the experiment. Note the appearance of dim fluorescence along axons (arrowheads). $\boldsymbol{F}$, Time lapse of PAGFP:Bsn953938 following restricted photoactivation shown in D. Note the appearance of photoactivated PAGFP:Bsn95-3938 at presynaptic sites residing outside of the photoactivated region (arrow and arrowhead). Also apparent is the significant reduction in fluorescence of all photoactivated boutons, probably attributable to a combination of photobleaching caused by recurrent imaging and the ongoing exchange of photoactivated PAGFP:Bsn95-3938 with nonphotoactivated PAGFP:Bsn95-3938 from sources outside the photoactivated region. and incorporation of newly synthesized protein. However, our photoactivation experiments (Fig. 5) indicate that Bassoon, in common with other presynaptic and postsynaptic molecules such as Synapsin I, ProSAP2, and PSD-95 (Gray et al., 2006; Tsuriel et al., 2006), can migrate from one synaptic locus to another and assimilate into the synaptic matrices at these sites, strengthening the notion that nearby synapses share, and perhaps compete over, local pools of such molecules (for review, see Staras, 2007). Interestingly, a retrospective immunohistochemical analysis of migrating SV packets (Krueger et al., 2003) (see also Tao-Cheng, 2007) indicates that Bassoon might migrate between nearby synapses in the form of transport packets containing unitary amounts of AZ material and SVs ("orphan release sites"). Our observations of small mobile PAGFP:Bsn953938 puncta migrating between synapses is consistent this mode of material exchange, as are the stepwise increases in fluorescence we observed in some FRAP experiments (supplemental Fig. 1, available at www.jneurosci.org as supplemental material). It is not clear to us, however, that this exchange mode is exclusive. We did observe relatively rapid and monotonous fluorescence recovery in some FRAP experiments, which could point to the existence of small pools of soluble Bassoon. In this respect, it is worth noting that photoactivation also exposed what seems to be dim diffuse axonal fluorescence (Figs. $4 B$, $5 E$ ), which could represent pools of soluble PAGFP:Bsn95-3938 or numerous, very small clusters of this protein.

In mice deficient for Bassoon, ribbon complexes of retinal photoreceptor synapses lose their anchoring to the presynaptic membrane (Dick et al., 2003). Conversely, presynaptic structure of glutamatergic synapses is not grossly affected, yet a large fraction of these are functionally inactive (Altrock et al., 2003). Experiments in which RNA interference was used to suppress Bassoon or Piccolo expression reveal that neither are essential for synapse formation but indicate that Piccolo (but not Bassoon) is involved in SV retention and mobilization (Leal-Ortiz et al., 2008). At present, this and other data provide tantalizing, yet not necessarily coherent, hints as to the role of the CAZ and its components in maintaining presynaptic organization and functional characteristics. We expect that future experiments in which presynaptic tenacity will be explored over longer timescales (days, weeks) in wild-type and genetically perturbed neurons will clarify the relation- 
ships between the CAZ, its composition, and presynaptic tenacity.

\section{References}

Altrock WD, tom Dieck S, Sokolov M, Meyer AC, Sigler A, Brakebusch C, Fässler R, Richter K, Boeckers TM, Potschka H, Brandt C, Löscher W, Grimberg D, Dresbach T, Hempelmann A, Hassan H, Balschun D, Frey JU, Brandstätter JH, Garner CC, et al. (2003) Functional inactivation of a fraction of excitatory synapses in mice deficient for the active zone protein bassoon. Neuron 37:787-800.

Betz A, Ashery U, Rickmann M, Augustin I, Neher E, Südhof TC, Rettig J, Brose N (1998) Munc13-1 is a presynaptic phorbol ester receptor that enhances neurotransmitter release. Neuron 21:123-136.

Bresler T, Shapira M, Boeckers T, Dresbach T, Futter M, Garner CC, Rosenblum K, Gundelfinger ED, Ziv NE (2004) Postsynaptic density assembly is fundamentally different from presynaptic active zone assembly. J Neurosci 24:1507-1520.

Chi P, Greengard P, Ryan TA (2001) Synapsin dispersion and reclustering during synaptic activity. Nat Neurosci 4:1187-1193.

Chi P, Greengard P, Ryan TA (2003) Synaptic vesicle mobilization is regulated by distinct synapsin I phosphorylation pathways at different frequencies. Neuron 38:69-78.

Darcy KJ, Staras K, Collinson LM, Goda Y (2006) Constitutive sharing of recycling synaptic vesicles between presynaptic boutons. Nat Neurosci 9:315-321.

De Paola V, Holtmaat A, Knott G, Song S, Wilbrecht L, Caroni P, Svoboda K (2006) Cell type-specific structural plasticity of axonal branches and boutons in the adult neocortex. Neuron 49:861-875.

Dick O, tom Dieck S, Altrock WD, Ammermüller J, Weiler R, Garner CC, Gundelfinger ED, Brandstätter JH (2003) The presynaptic active zone protein bassoon is essential for photoreceptor ribbon synapse formation in the retina. Neuron 37:775-786.

Dresbach T, Hempelmann A, Spilker C, tom Dieck S, Altrock WD, Zuschratter W, Garner CC, Gundelfinger ED (2003) Functional regions of the presynaptic cytomatrix protein bassoon: significance for synaptic targeting and cytomatrix anchoring. Mol Cell Neurosci 23:279-291.

Dresbach T, Torres V, Wittenmayer N, Altrock WD, Zamorano P, Zuschratter W, Nawrotzki R, Ziv NE, Garner CC, Gundelfinger ED (2006) Assembly of active zone precursor vesicles: obligatory trafficking of presynaptic cytomatrix proteins Bassoon and Piccolo via a trans-Golgi compartment. J Biol Chem 281:6038-6047.

Fenster SD, Chung WJ, Zhai R, Cases-Langhoff C, Voss B, Garner AM, Kaempf U, Kindler S, Gundelfinger ED, Garner CC (2000) Piccolo, a presynaptic zinc finger protein structurally related to bassoon. Neuron 25:203-214.

Fernández-Alfonso T, Kwan R, Ryan TA (2006) Synaptic vesicles interchange their membrane proteins with a large surface reservoir during recycling. Neuron 51:179-186.

Geracitano R, Kaufmann WA, Szabo G, Ferraguti F, Capogna M. (2007) Synaptic heterogeneity between mouse paracapsular intercalated neurons of the amygdala. J Physiol 585:117-134.

Gray NW, Weimer RM, Bureau I, Svoboda K (2006) Rapid redistribution of synaptic PSD-95 in the neocortex in vivo. PLoS Biol 4:2065-2075.

Harata N, Ryan TA, Smith SJ, Buchanan J, Tsien RW (2001) Visualizing recycling synaptic vesicles in hippocampal neurons by FM 1-43 photoconversion. Proc Natl Acad Sci U S A 98:12748-12753.

Husi H, Ward MA, Choudhary JS, Blackstock WP, Grant SG (2000) Proteomic analysis of NMDA receptor-adhesion protein signaling complexes. Nat Neurosci 3:661-669.

Kalla S, Stern M, Basu J, Varoqueaux F, Reim K, Rosenmund C, Ziv NE, Brose N (2006) Molecular dynamics of a presynaptic active zone protein studied in Munc13-1-enhanced yellow fluorescent protein knock-in mutant mice. J Neurosci 26:13054-13066.
Kraszewski K, Daniell L, Mundigl O, De Camilli P (1996) Mobility of synaptic vesicles in nerve endings monitored by recovery from photobleaching of synaptic vesicle-associated fluorescence. J Neurosci 16:5905-5913.

Krueger SR, Kolar A, Fitzsimonds RM (2003) The presynaptic release apparatus is functional in the absence of dendritic contact and highly mobile within isolated axons. Neuron 40:945-957.

Leal-Ortiz S, Waites CL, Terry-Lorenzo R, Zamorano P, Gundelfinger ED, Garner CC (2008) Piccolo modulation of Synapsinla dynamics regulates synaptic vesicle exocytosis. J Cell Biol 181:831-846.

Li Z, Murthy VN (2001) Visualizing postendocytic traffic of synaptic vesicles at hippocampal synapses. Neuron 31:593-605.

Murthy VN, Sejnowski TJ, Stevens CF (1997) Heterogeneous release properties of visualized individual hippocampal synapses. Neuron 18:599-612.

Patterson GH, Lippincott-Schwartz J (2002) A photoactivatable GFP for selective photolabeling of proteins and cells. Science 297:1873-1877.

Phillips GR, Huang JK, Wang Y, Tanaka H, Shapiro L, Zhang W, Shan WS, Arndt K, Frank M, Gordon RE, Gawinowicz MA, Zhao Y, Colman DR (2001) The presynaptic particle web: ultrastructure, composition, dissolution, and reconstitution. Neuron 32:63-77.

Ryan TA, Smith SJ (1995) Vesicle pool mobilization during action potential firing at hippocampal synapses. Neuron 14:983-989.

Sankaranarayanan S, Ryan TA (2000) Real-time measurements of vesicleSNARE recycling in synapses of the central nervous system. Nat Cell Biol 2:197-204

Sankaranarayanan S, Ryan TA (2001) Calcium accelerates endocytosis of vSNAREs at hippocampal synapses. Nat Neurosci 4:129-136.

Schoch S, Gundelfinger ED (2006) Molecular organization of the presynaptic active zone. Cell Tissue Res 326:379-391.

Shapira M, Zhai RG, Dresbach T, Bresler T, Torres VI, Gundelfinger ED, Ziv NE, Garner CC (2003) Unitary assembly of presynaptic active zones from Piccolo-Bassoon transport vesicles. Neuron 38:237-252.

Shepherd GM, Harris KM (1998) Three-dimensional structure and composition of $\mathrm{CA} 3 \rightarrow \mathrm{CA} 1$ axons in rat hippocampal slices: implications for presynaptic connectivity and compartmentalization. J Neurosci 18:8300-8310.

Staple JK, Morgenthaler F, Catsicas S (2000) Presynaptic heterogenity: vive la difference. News Physiol Sci 15:45-49.

Star EN, Newton AJ, Murthy VN (2005) Real-time imaging of Rab3a and Rab5a reveals differential roles in presynaptic function. J Physiol 569:103-117.

Staras K (2007) Share and share alike: trading of presynaptic elements between central synapses. Trends Neurosci 30:292-298.

Stettler DD, Yamahachi H, Li W, Denk W, Gilbert CD (2006) Axons and synaptic boutons are highly dynamic in adult visual cortex. Neuron 49:877-887.

Tao-Cheng JH (2006) Activity-related redistribution of presynaptic proteins at the active zone. Neuroscience 141:1217-1224.

Tao-Cheng JH (2007) Ultrastructural localization of active zone and synaptic vesicle proteins in a preassembled multi-vesicle transport aggregate Neuroscience 150:575-584.

tom Dieck S, Sanmartí-Vila L, Langnaese K, Richter K, Kindler S, Soyke A Wex H, Smalla KH, Kämpf U, Fränzer JT, Stumm M, Garner CC, Gundelfinger ED (1998) Bassoon, a novel zinc-finger CAG/glutaminerepeat protein selectively localized at the active zone of presynaptic nerve terminals. J Cell Biol 142:499-509.

Tsuriel S, Geva R, Zamorano P, Dresbach T, Boeckers T, Gundelfinger ED, Garner CC, Ziv NE (2006) Local sharing as a predominant determinant of synaptic matrix molecular dynamics. PLoS Biol 4:1572-1587.

Wienisch M, Klingauf J (2006) Vesicular proteins exocytosed and subsequently retrieved by compensatory endocytosis are nonidentical. Nat Neurosci 9:1019-1027. 